\title{
Association Between Tumor Mutation Burden and Prognosis in Lower-Grade Glioma: An Exploratory Study
}

\section{Di Cao}

Qiqihar Medical University

Jun Wang

Qiqihar Medical University

Yan Lin

Qiqihar Medical University

Guangwei Li ( $\square$ Igw@qmu.edu.cn )

Qiqihar Medical University

\section{Research Article}

Keywords: Lower-grade glioma, The Cancer Genome Atlas, Tumor mutation burden, TMB Prognostic Index, Tumor-infiltrating immune cells, Chinese Glioma Genome Atlas

Posted Date: January 14th, 2021

DOI: https://doi.org/10.21203/rs.3.rs-137165/v1

License: (c) (i) This work is licensed under a Creative Commons Attribution 4.0 International License. Read Full License 


\section{Abstract}

Background: The therapeutic efficacy of immune checkpoint inhibitor therapy is highly influenced by tumor mutation burden (TMB). The relationship between TMB and prognosis in lower-grade glioma is still unclear. We aimed to explore the relationships and mechanisms between them in lower-grade glioma.

Methods: We leveraged somatic mutation data from The Cancer Genome Atlas (TCGA). Clinical cases were divided into high- and low-TMB groups based on the median of TMB. Infiltrating immune cells were analyzed using CIBERSORT and differential expression analysis between the prognostic groups performed. The key genes were identified as intersecting between immune-related genes. Cox regression and survival analysis were performed on the intersecting genes. A total of 7 hub genes were identified. The effect of somatic copy number alterations (SCNA) of the hub genes on immune cell infiltration was analyzed using TIMER, which was used to determine the risk factors and immune infiltration status in LGG. Subsequently, based on hub genes, a TMB Prognosis Index (TMBPI) model was constructed to predict the risk in LGG patients. Besides, this model was validated using data from TCGA and Chinese Glioma Genome Atlas (CGGA).

Results: High-TMB favored worse prognosis (Pख0.001) and macrophage infiltration was an independent risk factor ( $P \otimes 0.001)$. In the high-TMB group $(P=0.033, P=0.009)$, the proportion of macrophages $\mathrm{M} 0$ and $\mathrm{M} 2$ increased and monocytes decreased $(\mathrm{P}=0.006)$. Besides, the SCNA of the hub genes affected the level of immune cell infiltration by varying degrees among which IGF2BP3, NPNT, and PLA2G2A had a significant impact. The AUC of the ROC curve at 1-, 3- and 5-years were all above 0.74.

Conclusions: This study implies that high-TMB correlated with unfavorable prognosis in lower-grade glioma. And high-TMB may have an impact on prognosis by changing tumor microenvironment, caused by the SCNAs of genes. The TMBPI model accurately predicted prognosis in LGG patients.

\section{Background}

Gliomas derived from neuroglial cells are the most common primary malignant central nervous system tumors. According to the World Health Organization (WHO) classification of gliomas based on histological grading, gliomas are graded as lower-grade glioma (LGG) include diffuse lower-grade and intermediate-grade gliomas ([WHO] grade II and III), astrocytoma, oligodendrogliomas, and oligoastrocytomas ${ }^{[1,2]}$. Although the prognosis of LGG patients is relatively favorable, LGG tends to progress rapidly into high-grade glioma and is characterized by high mortality rate. Common treatments for LGG include neurosurgical resection, watchful waiting, radiotherapy, and chemotherapy ${ }^{[3]}$. The emergence of immune checkpoint inhibitors has revolutionized the treatment of patients with advancedstage cancers ${ }^{[4]}$. However, the clinical efficacy of immunotherapy is dependent on multiple factors. TMB and tumor microenvironment (TME) have emerged as research hotspots recently. TMB is defined as the number of aberrant nucleotide sequences a given tumor may harbor has been shown to influence the 
efficacy of immunotherapy. Wang found that the proliferative activity and immune response were activated in high-TMB glioma ${ }^{[5]}$.

Furthermore, Gorsi reported that immune checkpoint inhibitors (Nivolumab) performed better in brain tumor patients with higher TMB ${ }^{[6]}$. Besides, crosstalk between neoplastic and immune cells in the TME also influences the progression of disease in humans ${ }^{[7]}$. Accumulating evidence shows that TMB and TME can be exploited to predict the clinical outcomes and therapeutic responses of the tumor.

These recent developments make immunotherapy increasingly valuable for LGG patients. Meanwhile, enhancing the efficacy of immunotherapy needs to be further explored to improve personalized therapeutic care. Therefore, in this study, data from the TCGA, a public database, was obtained to explore the relationship between TMB and prognosis in lower-grade glioma and try to explain the reasons why TMB can influence prognosis from the perspective of TME. Besides, a TMBPI model was constructed using TMB and gene expression profiles, to potentially improve patient survival and guide personalized therapy for LGG patients with better prognosis prediction. The TMBPI model was validated using both TCGA and CGGA databases.

\section{Results}

\section{Overview of mutation profiles.}

A total of 508 mutation profiles were downloaded from TCGA. The 'maftools' package was used to visualize the results based on the mutation data. The waterfall plot was used to display the mutation information including the name and frequency of mutant genes, different colors were used to represent different mutation types (Figure 2).

The most common mutation type was 'Missense Mutation' and SNP was the most frequent variant. C>T, $\mathrm{C}>\mathrm{A}$, and $\mathrm{T}>\mathrm{C}$ ranked in the top three of the SNV class, the median variants per sample was 25 . Moreover, the top ten mutated genes in LGG ranked with percentage included IDH1(77\%), TP53(46\%), ATRX(33\%), CIC (20\%), TTN (12\%), FUBP1(9\%), MUC16(6\%), NOTCH1(7\%), PIK3CA (7\%), EGFR (6\%) (Figure 3A-F).

And their gene symbols were shown in the gene cloud (Figure S1). Besides, a significant interaction of mutant genes (co-occurrence and mutual exclusion) was exhibited in the interaction plot, among which EGFR-IDH1, CIC-TP53 showed the most mutually exclusive relationships and ATRX-TP53 showed most co-occurrence relationships. (Figure $\mathbf{3 G}$ )

\section{High-TMB is associated with unfavorable prognosis.}

Clinical data of 513 LGG patients were included in this study. The clinicopathological parameters included age, sex, tumor grade, TMB level, and TMBPI (Table 1). Tables

Table 1. Parameters of 513 LGG patients from TCGA 


\begin{tabular}{|c|c|}
\hline Parameters & Number (\%) \\
\hline \multicolumn{2}{|l|}{ Vital status } \\
\hline Alive & $388(75.6)$ \\
\hline Dead & $125(24.4)$ \\
\hline \multicolumn{2}{|l|}{ Age } \\
\hline$\leq 45$ & $310(62.2)$ \\
\hline$>45$ & 203(37.8) \\
\hline \multicolumn{2}{|l|}{ Gender } \\
\hline Female & $289(56.3)$ \\
\hline Male & $284(43.7)$ \\
\hline \multicolumn{2}{|l|}{ Grade } \\
\hline Grade $\otimes$ & $248(48.3)$ \\
\hline Grade $\rrbracket$ & $264(51.5)$ \\
\hline Unknown & $1(0.2)$ \\
\hline \multicolumn{2}{|l|}{ TMB } \\
\hline High level & $241(47.0)$ \\
\hline Low level & $260(50.7)$ \\
\hline Undetected & $2(2.3)$ \\
\hline \multicolumn{2}{|l|}{ TMBPI } \\
\hline High level & $249(48.5)$ \\
\hline Low level & $250(48.7)$ \\
\hline Unknown & $14(2.8)$ \\
\hline
\end{tabular}

Patients were divided into two groups, high-TMB $(n=241)$ and low-TMB $(n=260)$ levels using the median TMB as the cutoff value. The survival analysis showed that the high-TMB group was associated with a worse prognosis compared with the low-TMB group (Pख0.001, Figure 4A).

Furthermore, box plots displayed that the TMB level was higher in older patients and grade III (Pख0.001) patients (Figure 4B, C). However, no significant differences were observed in associations of TMB with gender (Figure 4D).

Comparison of gene expression profiles between low-TMB and high-TMB groups 
A total of 394 DEGs were revealed after differential analysis (See supplementary material). Heatmap indicated that the expression level of genes in the high-TMB group (Figure 5A) was lower than in the low TMB group. GO enrichment analysis revealed that the DEGs were mainly associated with cell division and composition of the extracellular matrix (Figure 5C). Besides, KEGG analysis showed several enriched pathways (ECM-receptor interaction, human T-cell leukemia virus 1 infection, cell cycle, transcriptional misregulation in cancer, and P53 signaling pathway, etc.) (Figure 5D).

GSEA analysis revealed 26 pathways with the criterion of FDR<0.05. Some of the pathways were involved in mismatch repair, p53 signaling pathway, cell cycle, glutathione metabolism (Figure 5E). For details see supplementary materials for GO, KEGG, and GSEA analysis results.

\section{Differences in infiltration level of immune cells in TME}

The level of 6 types of immune cells in TME (B cells, CD4+ T cells, CD8+ T cells, Neutrophils, Macrophages, and Dendritic cells) was related to survival prognosis of LGG patients (Figure S2). Multivariable Cox proportional hazard model of TIMER model, which was constructed as Surv $(\mathrm{LGG}) \sim \mathrm{B}_{-}$ cell + CD8_Tcell + CD4_Tcell + Macrophage + Neutrophil + Dendritic, showed that high macrophage infiltration was an independent risk factor $(H R=1845.804, p=0.000$, Table 2).

Table 2. Multivariate Cox regression analysis of lower-grade glioma by TIMER database.

\begin{tabular}{|lllllll|}
\hline Cell types & coef & HR & 95\%Cl_I & 95\%Cl_u & P-value & sig \\
\hline B cell & 0.304 & 1.355 & 0.005 & 362.209 & 0.915 & - \\
\hline CD8 ${ }^{+}$Tcell & 6.076 & 435.270 & 0.523 & 362042.429 & 0.077 & - \\
\hline CD4 ${ }^{+}$Tcell & -3.956 & 0.019 & 0.000 & 33.325 & 0.299 & - \\
\hline Macrophage & 7.521 & 1845.804 & 44.941 & 75811.118 & 0.000 & $* \star *$ \\
\hline Neutrophil & -5.201 & 0.006 & 0.000 & 8.569 & 0.165 & - \\
\hline Dendritic & 2.136 & 8.469 & 0.202 & 355.685 & 0.263 & - \\
\hline
\end{tabular}

R square $=0.103(\max$ possible $=9.09 \mathrm{e}-01)$, Likelihood ratio test $\mathrm{p}=4.94 \mathrm{e}-10$, Wald test $\mathrm{p}=1.19 \mathrm{e}-12$ Score (log rank) test $p=1.4 \mathrm{e}-13$. P-value Significant Codes: $0 \leq * \star *<0.001 \leq * *<0.01 \leq *<0.05 \leq .<0.1$

Besides, based on the CIBERSORT algorithm, we obtained an estimation of the abundances of 22 immune cells in LGG, and results were visualized with bar plot. (Figure 6A).

Moreover, a comparison of the proportion of different immune cells and TMB levels (high-TMB vs. low$T M B)$ showed an increase in the proportion of macrophages M0 and M2 (P=0.033, P=0.009), and a decrease in the proportion of monocytes $(P=0.006$, Figure 6B).

Identification of 7 hub immune genes. 
The intersection was obtained between DEGs from high- and low-TMB groups and immune-related genes. A total of 24 genes were screened ( $|\log F C|>1.5, F D R<0.05)$ for further analysis (Figure 5B). To identify hub immune genes, the 24 genes were further screened by Cox regression and Kaplan-Meier analysis. Out of the 24 genes, 7 were considered hub TMB-related immune genes. They included HOXB4, IGF2BP3, IL 13RA2, NPNT, PLA2G2A, TNFAIP6, and WISP1. All p-values computed by Cox regression and KaplanMeier analysis were less than 0.05 (Figure 7A).

\section{Hub immune genes influence the infiltration of immune cells in TME.}

The SCNA module on the TIMER webserver was used to explore the relationship between the 7 hub immune genes and the infiltration of immune cells in TME. The results indicated that the two most common alterations were Arm-level Gain and Deep Deletion, and these had a significant effect on the level of immune cells in TME, especially PLA2G2A, IGF2BP3 and NPNT (Figure 7B). The rest were shown in Figure S3.

\section{Establishment and assessment of TMBPI model for LGG.}

To predict LGG patients' prognosis, the TMBPI model was established based on the 7 hub immune genes. Their expression data and coefficients were calculated by multivariate Cox regression analysis and the model was constructed as follows: $\mathrm{PI}=(0.248935 \times / G F 2 B P 3+0.045491 \times N P N T+0.073771 \times$ WISP1$0.245454 \times$ HOXB4 + 0.109997 $\times$ TNFAIP6+0.017986 $\times$ IL 13RA2 +0.025691 $\times P L A 2 G 2 A)$. Further, 499 out of 513 cases from the TCGA database were divided into high-risk $(n=249)$ and low-risk $(n=250)$ groups based on the median of TMBPI as the cutoff value. Besides, 413 cases from the CGGA database were divided into high-risk $(n=215)$ and low-risk $(n=216)$ groups. Compared with the low-risk group, the highrisk group was associated with poor prognosis (Pख0.001, Figure 8A-B). The ROC curves showed that the AUC at 1 - , 3- and 5-years overall survival (OS) prediction were $0.84,0.83$, and 0.73 , respectively for the TCGA database. Consistently, the AUC at $1-, 3$ - and 5-years were $0.75,0.75$, and 0.79 , respectively for the CGGA database (Figure 8C-D).

\section{Discussion}

The prognosis of LGG is closely related to genetic mutations. In this study, the most typical mutant gene in LGG was isocitrate dehydrogenase (IDH). There are two types of IDH, IDH1, and $I D H, I D H 1$ is strongly correlated with inflammation and immune response. Studies have shown that people with IDH1 mutations have a better prognosis ${ }^{[18,19]}$. Besides, mutations in ATRX and TP53 are also considered to be important factors driving tumor growth ${ }^{[20]}$. This necessitated the refinement of glioma subtypes in the 2016 WHO guidelines by inclusion of IDH1, TP53, and ATRX mutational analysis alongside an evaluation of $1 p / 19 q$ chromosomal codeletion [21]. This classification has revolutionized the treatment of gliomas with immunotherapy playing an important role. However, the efficacy in treating patients with an immune checkpoint inhibitor, such as PD-1 blockade, was various. The underlying mechanism remains to be explored deeply. TMB and immune cells in the TME are the focus of research. 
Recently, high TMB has been associated with improved patient response to immunotherapy, hence used as an important biomarker to guide immunotherapy ${ }^{[22,23]}$. Meanwhile, TMB was also related to prognosis and we found that high TMB suggested unfavored prognosis. It is particularly important to understand the mechanism of increased TMB. Studies have shown that many factors influence TMB. In this study, GSEA analysis revealed that the DNA mismatch repair (MMR) pathway may play an important role. Besides, other studies indicate that the loss of MMR may cause an increase in TMB levels. Mehdi's study indicated that two main pathways may be associated with the increase of TMB (hypermutation) in LGG; de novo pathway associated with constitutional defects in MMR genes and a post-treatment pathway. Besides, temozolomide was shown to induce damage in cells with MMR deficiency driving the acquisition of hypermutated populations without promoting a response to PD-1 blockade [24]. Moreover, immunohistochemistry for MMR serves as a screening test for hypermutated gliomas ${ }^{[25]}$. Therefore, existing evidence suggests that MMR deficiency can improve the formation of hypermutation which does no help or even worse to promote the efficacy of immunotherapy, especially when patients with that of deficiency received chemotherapy. It also shows that MMR detection should be applied to the diagnosis of LGG. Further studies are needed to clarify why high TMB causes poor prognosis. In this study, the TMB level was also related to the patient's age and was higher especially in older people. Similarly, there is evidence that WHO type II gliomas are more common in patients aged above 40 years old, which could be associated with increased TMB levels ${ }^{[26]}$.

Much research focus has been directed at understanding the components of TME in tumors. This has led to the revelation that interaction between tumor and immune cells defines the time-course of cancer by modulating tumor malignancy, immune cell infiltration, immune evasion and the efficacy of immunotherapy ${ }^{[27,28]}$. In this study, immune cell infiltration in LGG was related to prognosis, and macrophages found to be an independent risk factor.

Existing evidence indicates that infiltrating monocytes and macrophages are associated with the formation and evolution of gliomas. They can release mitogens and invasion-promoting factors to increase glioma cell proliferation and promote tumor cell migration. Moreover, they also elaborate on other factors that increase extracellular matrix degradation and promote cell invasion and motility of glioma ${ }^{[29]}$. However, in this study, the proportions of M2 and M0 macrophages in the high TMB group were enhanced compared with the low TMB group. A study by Vidyarthi indicated that high levels of M2 macrophages infiltration are characteristic in high-grade gliomas, and this implies a poor prognostic signature ${ }^{[30]}$. Besides, in our study, no significant differences were found in the proportion of $\mathrm{T}$ cells between high- and low-TMB groups. Meanwhile, Mehdi's study contrasted T cells infiltration between MMR-deficiency and MMR-proficient cells in colorectal cancer and gliomas. Results demonstrated that in gliomas $T$ cells infiltration was not significant, which in colorectal cancer was completely different. These results indicated and supported that TMB level could hardly have an impact on T cells infiltration. Further, it showed negative results on the association between TMB and efficacy of PD-1 blockade. However, we did not observe changes in PD-1 and PD-L1 expression levels and this needs to be further researched. In 
general, it is suggested that high TMB might may increase malignancy of lower-grade glioma by modifying the infiltration landscape of immune cells.

The 7 hub immune genes identified using the SCNA in this study, were shown to significantly affect the infiltration of immune cells in TME, especially IGF2BP3, NPNT, and PLA2G2A. Besides, high expression of these genes showed a worse prognosis. Evidence shows that SCNA of genes was tightly bound to cancer. In regions of focal SCNA, some tumor suppressor genes and oncogenes have been identified, however, larger arm-level SCNAs are difficult to determine the drivers ${ }^{[31]}$. Arm-level SCNAs are associated with poor survival outcomes such as the loss of chromosome $9 \mathrm{p}$ which harbors the tumor suppressor CDKN2A in LGG. In the present study, IGF2BP3, NPNT, and PLA2G2A all had either arm-level gain or arm-level deletion. Interestingly, in our study arm-level SCNAs have had effects on the level of immune cells in TME.

A study by Deng provided a list of prognostic immune-related genes associated with immune cell infiltration. Two of the genes in the 7 hub immune genes identified in our study, HOXB4 and PLA2G2A, also appear in the gene list by Deng ${ }^{[32]}$. Therefore, it can be speculated that the mutants of these genes may affect the immune cells in TME due to alterations in copy number, which increases TMB and makes clinical outcomes worse. Moreover, no study has reported on the specific role of PLA2G2A in the progression of glioma. Therefore, PLA2G2A may be worth to study further.

Based on the 7 hub immune genes, the TMBPI model was established and its accuracy tested using ROC curves for 1-, 3- and 5-years OS prediction with TCGA and CGGA databases. Results showed that the TMBPI model has good accuracy and can be used to predict the clinical outcomes and assess the risk levels for LGG patients.

These findings suggested high-TMB correlated with unfavorable prognosis in lower-grade glioma. And high-TMB may have an impact on prognosis through alterations of TME, was caused by the SCNAs of genes. The TMBPI model showed a good accuracy in terms of predicting prognosis in LGG patients. However, the validity and practicality of the TMBPI model remain to be validated. Further research is needed to clarify the underlying mechanism.

\section{Methods}

\section{Data Source}

Figure 1 shows this study's workflow diagram. LGG transcriptome data, single nucleotide variants (SNV) data, and clinical data were downloaded from TCGA (https://portal.gdc.cancer.gov/) in April 2020. In this study, the workflow type of transcriptome data was HTseq-FPKM, and mutation data type was 'Masked Somatic Mutation'. SNV was identified by the MuTect. Besides, the mRNAseq_693 dataset acquired from the CGGA (http://www.cgga.org.cn/index.jsp/) which is a Chinese public glioma database was selected and included 431 lower-grade glioma cases used to validate the TMBPI model (see part 2.6).

\section{Data Grouping and Processing}


SNV data was summarized using R package 'maftools' to determine the condition of somatic gene mutation. Perl scripts were used to calculate the mutation frequency based on the number of variants in each sample. For group analysis, LGG samples were divided into high and low TMB groups based on median TMB. Differentially expressed genes (DEGs) between the two groups were compared by 'limma' package and the thresholds were $\mathrm{p}$-value $<0.05$ and $|\log \mathrm{FC}|>1$. Heatmaps were constructed in $\mathrm{R}$ using the 'pheatmap' package to show the different gene expression profiles. Besides, survival information was merged with TMB data using Entrez ID to assess the survival divergence using Kaplan-Meier analysis. Pvalues were calculated by the Log-Rank test. Wilcoxon Rank-Sum Test was used to determine the relationship between TMB level and clinical characteristics.

\section{GO, KEGG and GSEA analysis}

Gene ontology (GO) analysis was performed for function annotation of DEGs using 'clusterProfiler', 'enrichplot','ggplot2' packages ${ }^{[8,9]}$. Two approaches were used to display significantly enriched pathways; Kyoto Encyclopedia of Genes and Genomes (KEGG) ${ }^{[10]}$ and Gene set enrichment analysis (GSEA). KEGG is also implemented in the R programming language. GSEA software (version 4.0.3) was used to show the enrichment outcomes ${ }^{[11,12]}$. The TMB level served as phenotype and gene sets database was 'c2.cp.kegg.v7.1.symbols.gmt [Curated]' which was from MSigDB database (https://www.gseamsigdb.org/gsea/index.jsp/) ${ }^{[13]}$. False discovery rate $($ FDR $)<0.05$ was considered evidence of significance.

\section{TIMER database and CIBERSORT analysis}

TIMER web server is a comprehensive resource for systematical analysis of immune infiltrates across diverse cancer types ${ }^{[14]}$. 'Survival' module was selected to explore the association between LGG clinical outcome and an abundance of immune infiltrates. TIMER was used to calculate the immune infiltration. The P-value of a log-rank test was shown for each plot. The Cox model of 'Survival' was fitted using function coxph() from R package 'survival'; The coefficient 'coef' reads as a regression coefficient. HR provides the hazard ratio, and its lower and upper $95 \%$ confidential interval was shown in $95 \% \mathrm{Cl} \_$I \& 95\%Cl_u. The SCNA module was used to explore the correlation between different SCNAs of hub TMBrelated immune genes and the abundance of immune infiltrates.

CIBERSORT is a deconvolution algorithm that uses a set of reference gene expression values (547 genes) to predict 22 immune cell type proportions from bulk tumor sample expression data using support vector regression ${ }^{[15]}$. CIBERSORT algorithm was used to calculate immune cell composition from LGG gene expression profiles and estimate the tumor-infiltrating immune cells in LGG. The Wilcoxon Rank-Sum test was employed to assess the differences in tumor-infiltrating immune cells between the high and low TMB groups. Visualization of differential infiltration level of immune cells was performed using 'vioplot' package with a p-value.

\section{Selection of hub TMB-related Immune genes}


A list of the immune-related genes was downloaded from the InnateDB database (https://www.innatedb.ca/) and a gene intersect obtained for DEGs in the two TMB groups using the 'VennDiagram' package ${ }^{[16]}$. The screening criteria were $|\log F C|>1.5$ and FDR $<0.05$. Survival Kaplan-Meier analysis was conducted via a 'for cycle' $\mathrm{R}$ script to explore the hub immune genes related to survival outcomes and P-value was shown in the plot.

\section{Construction of TMB Prognostic index (TMBPI) for hub immune genes}

Multivariate Cox regression analysis was conducted to obtain coefficients of 7 hub immune genes. The formula: was used to construct TMBPI ${ }^{[17]}$. The performance was determined with the receiver operating characteristic (ROC) curve and the area under the ROC curve (AUC) based on datasets from TCGA and the CGGA databases using the 'timeROC' R package. The LGG patients were divided into a high-risk group and low-risk group using the median prognostic index as the threshold. Kaplan-Meier survival method was conducted to compare the survival differences between the two groups. The differences between the groups were tested by the log rank test.

\section{Statistical analysis.}

Differences in survival were assessed using the Cox regression models ( $R$ package survival).

Normalization of data was conducted using the 'limma' package. Comparisons between two groups were performed using the non-parametric Wilcoxon rank-sum test. P-value $<0.05$ was considered statistically significant and all statistical analyses were performed in R software (v3.6.2).

\section{Conclusions}

Our study implied High-TMB favored worse prognosis in lower-grade glioma. Moreover, SCNAs of 7 immune TMB-related genes may have effects on the level of TMB and furtherly influence immune cells in TME resulting in unfavorable prognosis. The proportion of macrophages $\mathrm{M} 0$ and $\mathrm{M} 2$ in TME were increased in the high-TMB group, and monocytes decreased in lower-grade glioma. Last but not least, TMBPI model is an effective prognostic model to predict the risk of patients with lower-grade glioma.

\section{Abbreviations}

LGG, lower-grade glioma; TMB, tumor mutation burden; TMBPI, tumor mutation burden prognosis index; MMR, mismatch repair; SCNA, somatic copy number alterations; ROC, receiver operating characteristic; GO, Gene Ontology; KEGG, Kyoto Encyclopedia of Genes and Genomes; GSEA Gene Set Enrichment Analysis; TME, tumor microenvironment; DEGs, differential expression genes; TCGA, The Cancer Genome Atlas; CGGA, Chinese Glioma Genome Atlas.

\section{Declarations}

\section{Ethics approval and consent to participate}


Not applicable.

\section{Consent for publication}

Not applicable.

\section{Availability of data and materials}

All data used in this manuscript were from public data repositories. The lower-grade glioma data is available in the TCGA database: https://portal.gdc.cancer.gov/repository. The validation data of this study is available in the CGGA database: http://www.cgga.org.cn/download.jsp/. Immune-related genes list is available in the InnateDB database: https://innatedb.com/redirect.do?go=resourcesGeneLists.

\section{Competing interests}

The authors declare that they have no competing interests.

\section{Funding}

The study was supported by item of Scientific Research Fund for Doctor of Qiqihar Medical University (No. QY2016B-16) and the National College Students Innovation and Entrepreneurship Training Program (CN) (No.201711230020)

\section{Author's contributions}

$C D, L G W$ and $L Y$ initiated and designed the experiments. CD, WJ, LGW and $L Y$ performed the experiments and collected the data. CD analyzed the data and wrote the main manuscript; WJ, LGW and LY revised the manuscript. The authors read and approved the final manuscript.

\section{Acknowledgements}

Not applicable.

\section{References}

1. D.N. Louis, H. Ohgaki, O.D. Wiestler, W.K. Cavenee, P.C. Burger, A. Jouvet, B.W. Scheithauer, P. Kleihues, The 2007 WHO classification of tumours of the central nervous system, Acta Neuropathol. 114 (2007) 97-109, https://doi.org/10.1007/s00401-007-0243-4.

2. Q.T. Ostrom, H. Gittleman, P. Farah, A. Ondracek, Y. Chen, Y. Wolinsky, N.E. Stroup, C. Kruchko, J.S. Barnholtz-Sloan, CBTRUS statistical report: Primary brain and central nervous system tumors diagnosed in the United States in 2006-2010, Neuro-oncology 15 Suppl 2 (2013) ii1-56, https://doi.org/10.1093/neuonc/not151.

3. Y. Wang, B. Zhao, W. Chen, L. Liu, W. Chen, L. Zhou, Z. Kong, C. Dai, Y. Wang, W. Ma, Pretreatment Geriatric Assessments of Elderly Patients with Glioma: Development and Implications, Aging Dis 11 
(2020) 448-461, https://doi.org/10.14336/AD.2019.0527.

4. M.K. Callahan, M.A. Postow, J.D. Wolchok, Targeting T Cell Co-receptors for Cancer Therapy, Immunity 44 (2016) 1069-1078, https://doi.org/10.1016/j.immuni.2016.04.023.

5. L. Wang, J. Ge, Y. Lan, Y. Shi, Y. Luo, Y. Tan, M. Liang, S. Deng, X. Zhang, W. Wang, Y. Tan, Y. Xu, T. Luo, Tumor mutational burden is associated with poor outcomes in diffuse glioma, BMC Cancer 20 (2020) 213, https://doi.org/10.1186/s12885-020-6658-1.

6. H.S. Gorsi, D.M. Malicki, V. Barsan, M. Tumblin, L. Yeh-Nayre, M. Milburn, J.D. Elster, J.R. Crawford, Nivolumab in the Treatment of Recurrent or Refractory Pediatric Brain Tumors: A Single Institutional Experience, J. Pediatr. Hematol. Oncol. 41 (2019) e235-235e241, https://doi.org/10.1097/MPH.0000000000001339.

7. J.K. Bujak, I.M. Szopa, R. Pingwara, O. Kruczyk, N. Krzemińska, J. Mucha, K. Majchrzak-Kuligowska, The Expression of Selected Factors Related to T Lymphocyte Activity in Canine Mammary Tumors, Int J Mol Sci 21 (2020) , https://doi.org/10.3390/ijms21072292.

8. M. Ashburner, C.A. Ball, J.A. Blake, D. Botstein, H. Butler, J.M. Cherry, A.P. Davis, K. Dolinski, S.S. Dwight, J.T. Eppig, M.A. Harris, D.P. Hill, L. Issel-Tarver, A. Kasarskis, S. Lewis, J.C. Matese, J.E. Richardson, M. Ringwald, G.M. Rubin, G. Sherlock, Gene ontology: tool for the unification of biology. The Gene Ontology Consortium, Nat. Genet. 25 (2000) 25-29, https://doi.org/10.1038/75556.

9. The Gene Ontology Consortium, The Gene Ontology Resource: 20 years and still GOing strong, Nucleic Acids Res. 47 (2019) D330-330D338, https://doi.org/10.1093/nar/gky1055.

10. M. Kanehisa, S. Goto, KEGG: kyoto encyclopedia of genes and genomes, Nucleic Acids Res. 28 (2000) 27-30, https://doi.org/10.1093/nar/28.1.27.

11. V.K. Mootha, C.M. Lindgren, K.F. Eriksson, A. Subramanian, S. Sihag, J. Lehar, P. Puigserver, E. Carlsson, M. Ridderstråle, E. Laurila, N. Houstis, M.J. Daly, N. Patterson, J.P. Mesirov, T.R. Golub, P. Tamayo, B. Spiegelman, E.S. Lander, J.N. Hirschhorn, D. Altshuler, L.C. Groop, PGC-1alpha-responsive genes involved in oxidative phosphorylation are coordinately downregulated in human diabetes, Nat. Genet. 34 (2003) 267-273, https://doi.org/10.1038/ng1180.

12. A. Subramanian, P. Tamayo, V.K. Mootha, S. Mukherjee, B.L. Ebert, M.A. Gillette, A. Paulovich, S.L. Pomeroy, T.R. Golub, E.S. Lander, J.P. Mesirov, Gene set enrichment analysis: a knowledge-based approach for interpreting genome-wide expression profiles, Proc. Natl. Acad. Sci. U.S.A. 102 (2005) 15545-15550, https://doi.org/10.1073/pnas.0506580102.

13. A. Liberzon, C. Birger, H. Thorvaldsdóttir, M. Ghandi, J.P. Mesirov, P. Tamayo, The Molecular Signatures Database (MSigDB) hallmark gene set collection, Cell Syst 1 (2015) 417-425, https://doi.org/10.1016/j.cels.2015.12.004.

14. T. Li, J. Fan, B. Wang, N. Traugh, Q. Chen, J.S. Liu, B. Li, X.S. Liu, TIMER: A Web Server for Comprehensive Analysis of Tumor-Infiltrating Immune Cells, Cancer Res. 77 (2017) e108-108e110, https://doi.org/10.1158/0008-5472.CAN-17-0307.

15. A.M. Newman, C.L. Liu, M.R. Green, A.J. Gentles, W. Feng, Y. Xu, C.D. Hoang, M. Diehn, A.A. Alizadeh, Robust enumeration of cell subsets from tissue expression profiles, Nat. Methods 12 (2015) 453-457, 
https://doi.org/10.1038/nmeth.3337.

16. K. Breuer, A.K. Foroushani, M.R. Laird, C. Chen, A. Sribnaia, R. Lo, G.L. Winsor, R.E. Hancock, F.S.

Brinkman, D.J. Lynn, InnateDB: systems biology of innate immunity and beyond-recent updates and continuing curation, Nucleic Acids Res. 41 (2013) D1228-1233, https://doi.org/10.1093/nar/gks1147.

17. C. Zhang, Z. Li, F. Qi, X. Hu, J. Luo, Exploration of the relationships between tumor mutation burden with immune infiltrates in clear cell renal cell carcinoma, Ann Transl Med 7 (2019) 648, https://doi.org/10.21037/atm.2019.10.84.

18. M. Ceccarelli, F.P. Barthel, T.M. Malta, T.S. Sabedot, S.R. Salama, B.A. Murray, O. Morozova, Y. Newton, A. Radenbaugh, S.M. Pagnotta, S. Anjum, J. Wang, G. Manyam, P. Zoppoli, S. Ling, A.A. Rao, M. Grifford, A.D. Cherniack, H. Zhang, L. Poisson, C.G. Carlotti Jr, D.P. Tirapelli, A. Rao, T. Mikkelsen, C.C. Lau, W.K. Yung, R. Rabadan, J. Huse, D.J. Brat, N.L. Lehman, J.S. Barnholtz-Sloan, S. Zheng, K. Hess, G. Rao, M. Meyerson, R. Beroukhim, L. Cooper, R. Akbani, M. Wrensch, D. Haussler, K.D. Aldape, P.W. Laird, D.H. Gutmann, TCGA Research Network, H. Noushmehr, A. lavarone, R.G. Verhaak, Molecular Profiling Reveals Biologically Discrete Subsets and Pathways of Progression in Diffuse Glioma, Cell 164 (2016) 550-563, https://doi.org/10.1016/j.cell.2015.12.028.

19. R. Otani, T. Uzuka, F. Higuchi, H. Matsuda, M. Nomura, S. Tanaka, A. Mukasa, K. Ichimura, P. Kim, K. Ueki, IDH-mutated astrocytomas with 19q-loss constitute a subgroup that confers better prognosis, Cancer Sci. 109 (2018) 2327-2335, https://doi.org/10.1111/cas.13635.

20. X.Y. Liu, N. Gerges, A. Korshunov, N. Sabha, D.A. Khuong-Quang, A.M. Fontebasso, A. Fleming, D. Hadjadj, J. Schwartzentruber, J. Majewski, Z. Dong, P. Siegel, S. Albrecht, S. Croul, D.T. Jones, M. Kool, M. Tonjes, G. Reifenberger, D. Faury, G. Zadeh, S. Pfister, N. Jabado, Frequent ATRX mutations and loss of expression in adult diffuse astrocytic tumors carrying IDH1/IDH2 and TP53 mutations, Acta Neuropathol. 124 (2012) 615-625, https://doi.org/10.1007/s00401-012-1031-3.

21. D.N. Louis, A. Perry, G. Reifenberger, A. von Deimling, D. Figarella-Branger, W.K. Cavenee, H. Ohgaki, O.D. Wiestler, P. Kleihues, D.W. Ellison, The 2016 World Health Organization Classification of Tumors of the Central Nervous System: a summary, Acta Neuropathol. 131 (2016) 803-820, https://doi.org/10.1007/s00401-016-1545-1.

22. D.T. Le, J.N. Uram, H. Wang, B.R. Bartlett, H. Kemberling, A.D. Eyring, A.D. Skora, B.S. Luber, N.S. Azad, D. Laheru, B. Biedrzycki, R.C. Donehower, A. Zaheer, G.A. Fisher, T.S. Crocenzi, J.J. Lee, S.M. Duffy, R.M. Goldberg, A. de la Chapelle, M. Koshiji, F. Bhaijee, T. Huebner, R.H. Hruban, L.D. Wood, N. Cuka, D.M. Pardoll, N. Papadopoulos, K.W. Kinzler, S. Zhou, T.C. Cornish, J.M. Taube, R.A. Anders, J.R. Eshleman, B. Vogelstein, L.A. Diaz Jr, PD-1 Blockade in Tumors with Mismatch-Repair Deficiency, N. Engl. J. Med. 372 (2015) 2509-2520, https://doi.org/10.1056/NEJMoa1500596.

23. M. Yarchoan, A. Hopkins, E.M. Jaffee, Tumor Mutational Burden and Response Rate to PD-1 Inhibition, N. Engl. J. Med. 377 (2017) 2500-2501, https://doi.org/10.1056/NEJMc1713444.

24. M. Touat, Y.Y. Li, A.N. Boynton, L.F. Spurr, J.B. Iorgulescu, C.L. Bohrson, I. Cortes-Ciriano, C. Birzu, J.E. Geduldig, K. Pelton, M.J. Lim-Fat, S. Pal, R. Ferrer-Luna, S.H. Ramkissoon, F. Dubois, C. Bellamy, N. 
Currimjee, J. Bonardi, K. Qian, P. Ho, S. Malinowski, L. Taquet, R.E. Jones, A. Shetty, K.H. Chow, R. Sharaf, D. Pavlick, L.A. Albacker, N. Younan, C. Baldini, M. Verreault, M. Giry, E. Guillerm, S. Ammari, F. Beuvon, K. Mokhtari, A. Alentorn, C. Dehais, C. Houillier, F. Laigle-Donadey, D. Psimaras, E.Q. Lee, L. Nayak, J.R. McFaline-Figueroa, A. Carpentier, P. Cornu, L. Capelle, B. Mathon, J.S. Barnholtz-Sloan, A. Chakravarti, W.L. Bi, E.A. Chiocca, K.P. Fehnel, S. Alexandrescu, S.N. Chi, D. Haas-Kogan, T.T. Batchelor, G.M. Frampton, B.M. Alexander, R.Y. Huang, A.H. Ligon, F. Coulet, J.Y. Delattre, K. HoangXuan, D.M. Meredith, S. Santagata, A. Duval, M. Sanson, A.D. Cherniack, P.Y. Wen, D.A. Reardon, A. Marabelle, P.J. Park, A. Idbaih, R. Beroukhim, P. Bandopadhayay, F. Bielle, K.L. Ligon, Mechanisms and therapeutic implications of hypermutation in gliomas, Nature 580 (2020) 517-523, https://doi.org/10.1038/s41586-020-2209-9.

25. M. McCord, A. Steffens, R. Javier, K.L. Kam, K. McCortney, C. Horbinski, The efficacy of DNA mismatch repair enzyme immunohistochemistry as a screening test for hypermutated gliomas, Acta Neuropathol Commun 8 (2020) 15, https://doi.org/10.1186/s40478-020-0892-2.

26. R.S. Youland, D.A. Schomas, P.D. Brown, C. Nwachukwu, J.C. Buckner, C. Giannini, I.F. Parney, N.N. Laack, Changes in presentation, treatment, and outcomes of adult low-grade gliomas over the past fifty years, Neuro-oncology 15 (2013) 1102-1110, https://doi.org/10.1093/neuonc/not080.

27. J. Gupta, H. Ahsan Safdari, M. Hoque, Nanoparticle mediated cancer immunotherapy, Semin. Cancer Biol. (2020) , https://doi.org/10.1016/j.semcancer.2020.03.015.

28. P. Sarode, M.B. Schaefer, F. Grimminger, W. Seeger, R. Savai, Macrophage and Tumor Cell Cross-Talk Is Fundamental for Lung Tumor Progression: We Need to Talk, Front Oncol 10 (2020) 324, https://doi.org/10.3389/fonc.2020.00324.

29. D.H. Gutmann, H. Kettenmann, Microglia/Brain Macrophages as Central Drivers of Brain Tumor Pathobiology, Neuron 104 (2019) 442-449, https://doi.org/10.1016/j.neuron.2019.08.028.

30. A. Vidyarthi, T. Agnihotri, N. Khan, S. Singh, M.K. Tewari, B.D. Radotra, D. Chatterjee, J.N. Agrewala, Predominance of M2 macrophages in gliomas leads to the suppression of local and systemic immunity, Cancer Immunol. Immunother. 68 (2019) 1995-2004, https://doi.org/10.1007/s00262-01902423-8.

31. D.M. Roy, L.A. Walsh, A. Desrichard, J.T. Huse, W. Wu, J. Gao, P. Bose, W. Lee, T.A. Chan, Integrated Genomics for Pinpointing Survival Loci within Arm-Level Somatic Copy Number Alterations, Cancer Cell 29 (2016) 737-750, https://doi.org/10.1016/j.ccell.2016.03.025.

32. X. Deng, D. Lin, X. Zhang, X. Shen, Z. Yang, L. Yang, X. Lu, L. Yu, N. Zhang, J. Lin, Profiles of immunerelated genes and immune cell infiltration in the tumor microenvironment of diffuse lower-grade gliomas, J. Cell. Physiol. (2020) , https://doi.org/10.1002/jcp.29633.

\section{Figures}




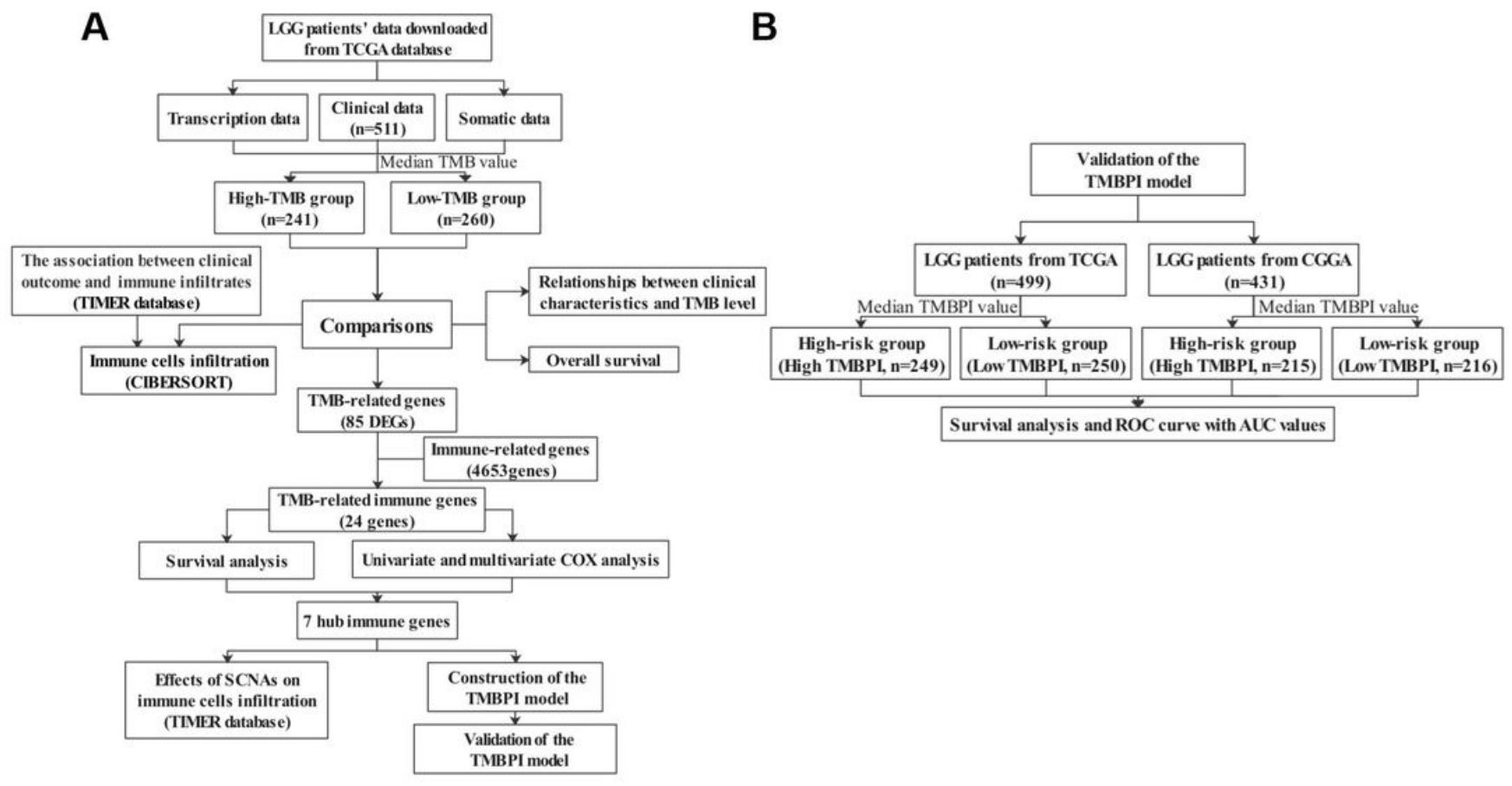

\section{Figure 1}

Work flow diagram. (A) Workflow of overall design. (B) Validation of TMBPI model with TCGA and CGGA databases. 
Altered in 491 (96.65\%) of 508 samples.



\section{Figure 2}

The waterfall plot shows the single most damaging variant found per gene and per sample in lower-grade glioma, indicating mutation types with various colors. 


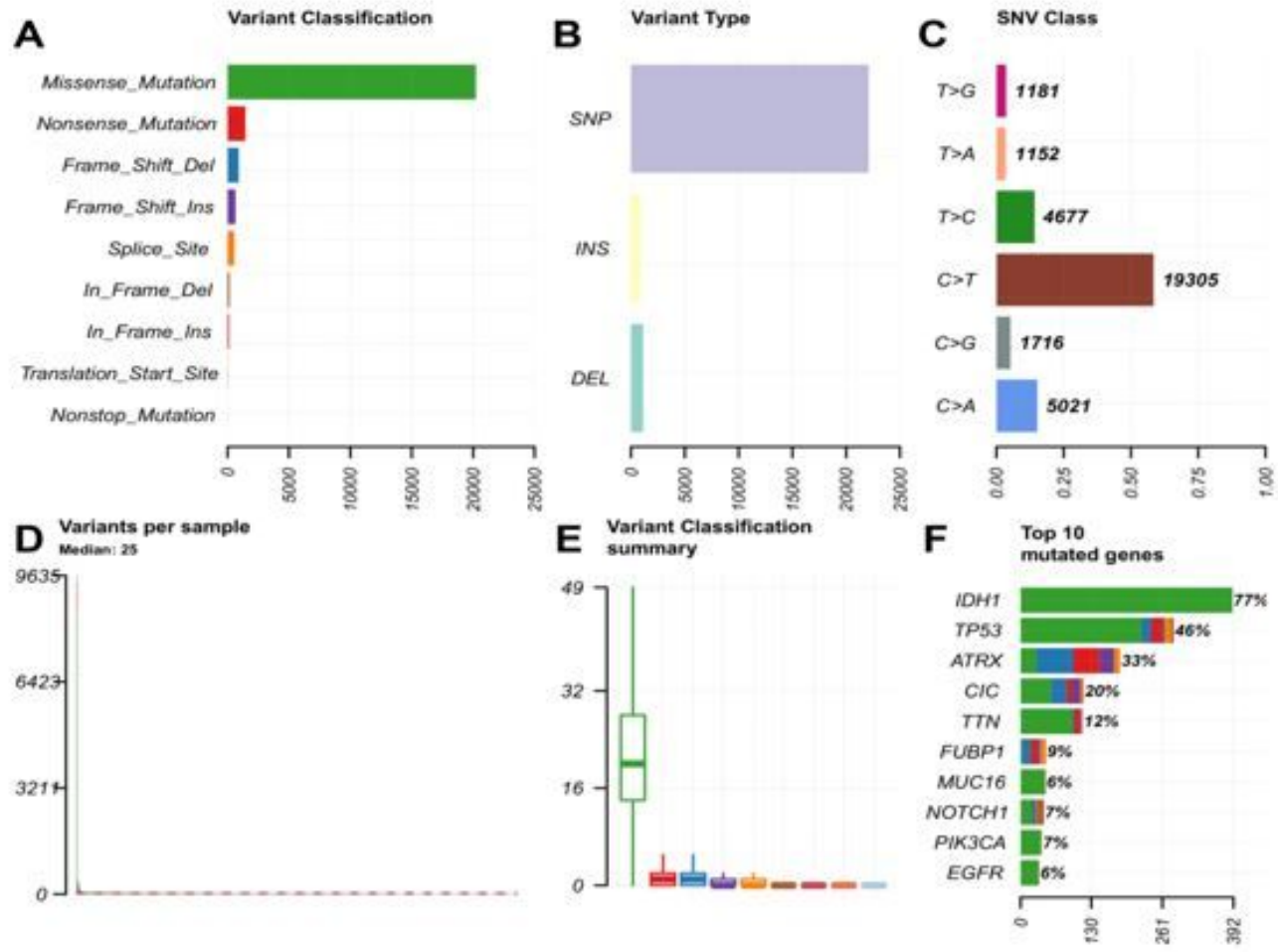

G

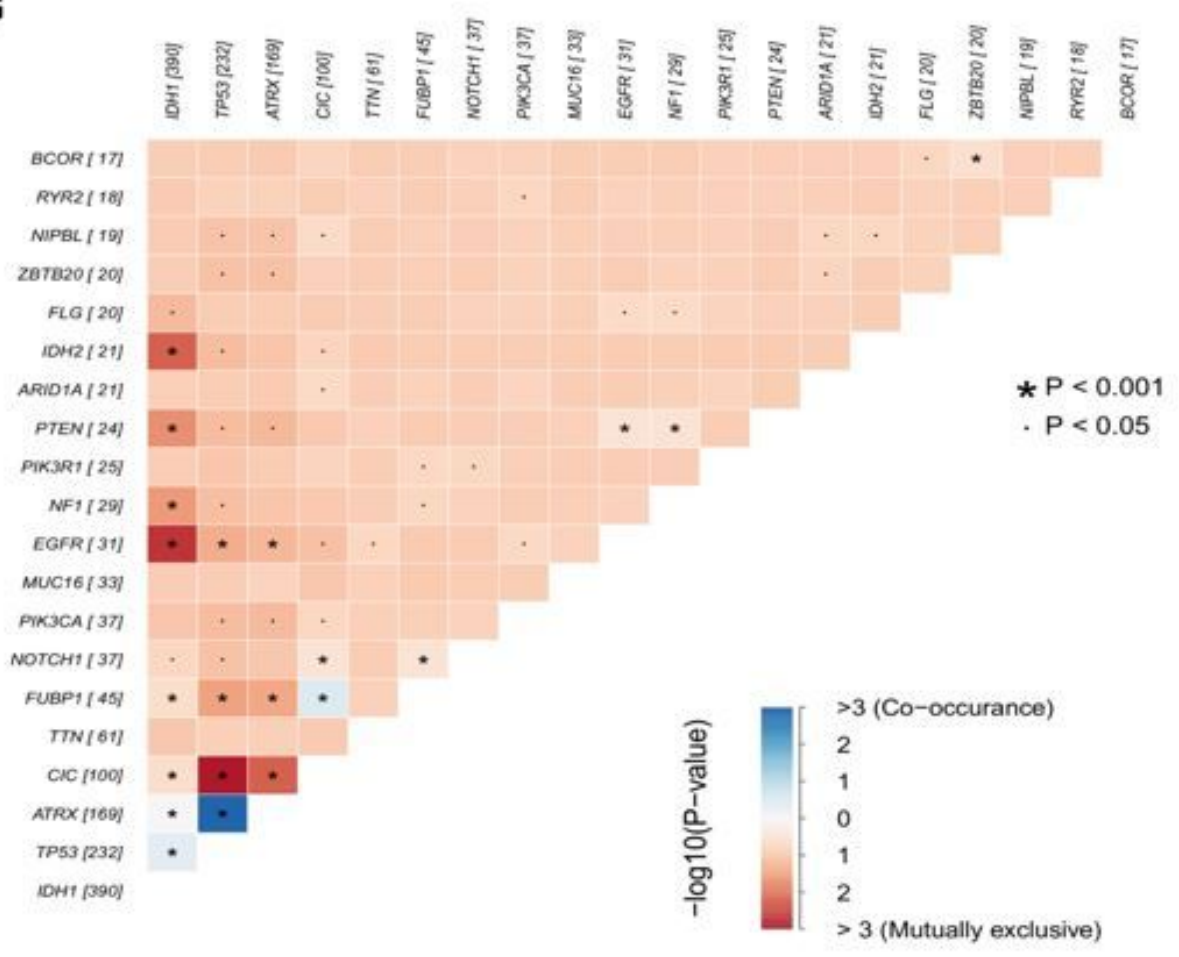

\section{Figure 3}

Summary of somatic mutations and gene interactions. (A-F) Summary of somatic mutations. (G) Interaction plot exhibits co-occurrence and mutually exclusive of mutation genes. 

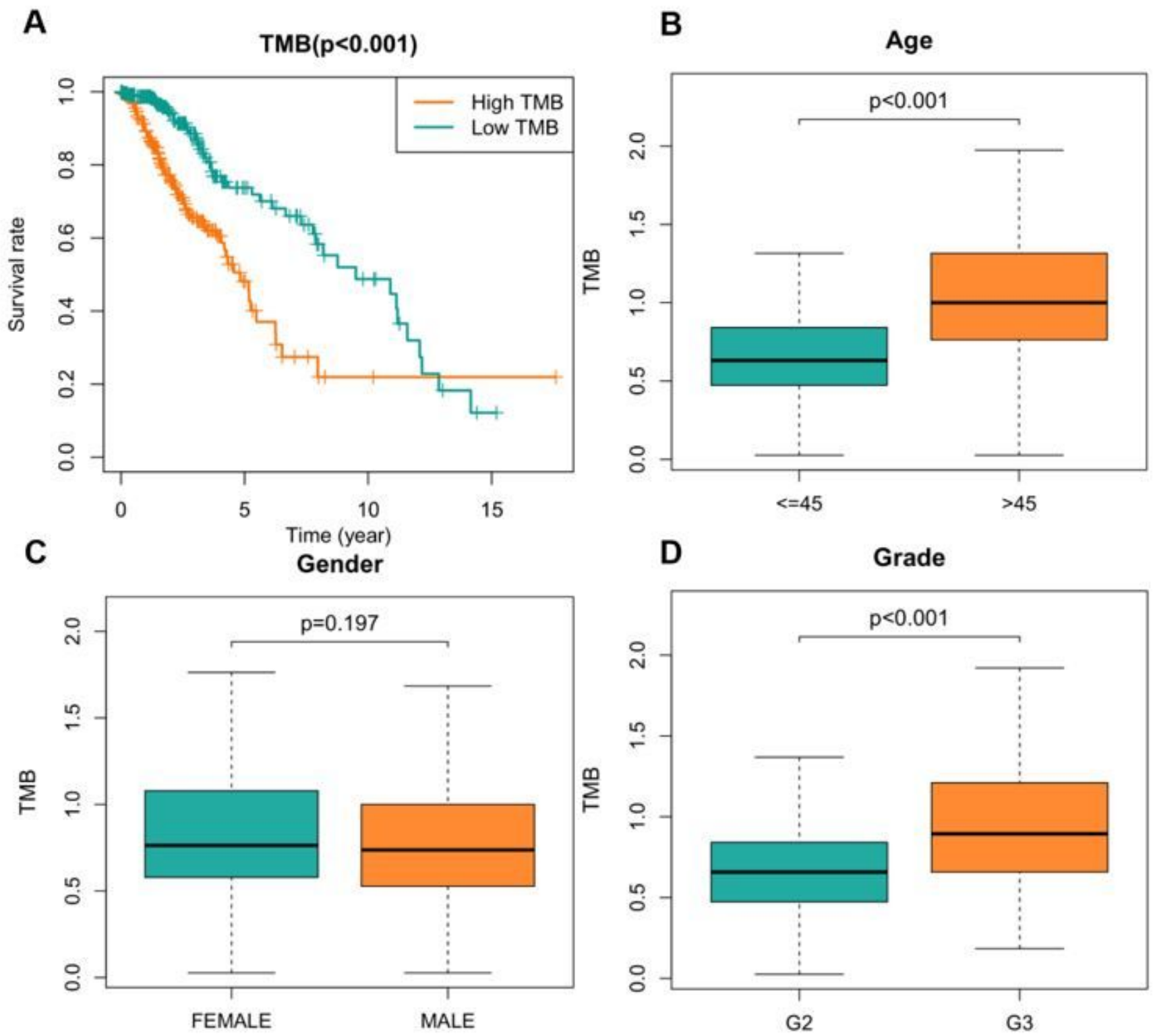

Figure 4

TMB and lower-grade glioma. (A) High TMB of lower-grade glioma was associated with poor overall survival. (B-D) Relationships between TMB level and clinicopathological parameters. 


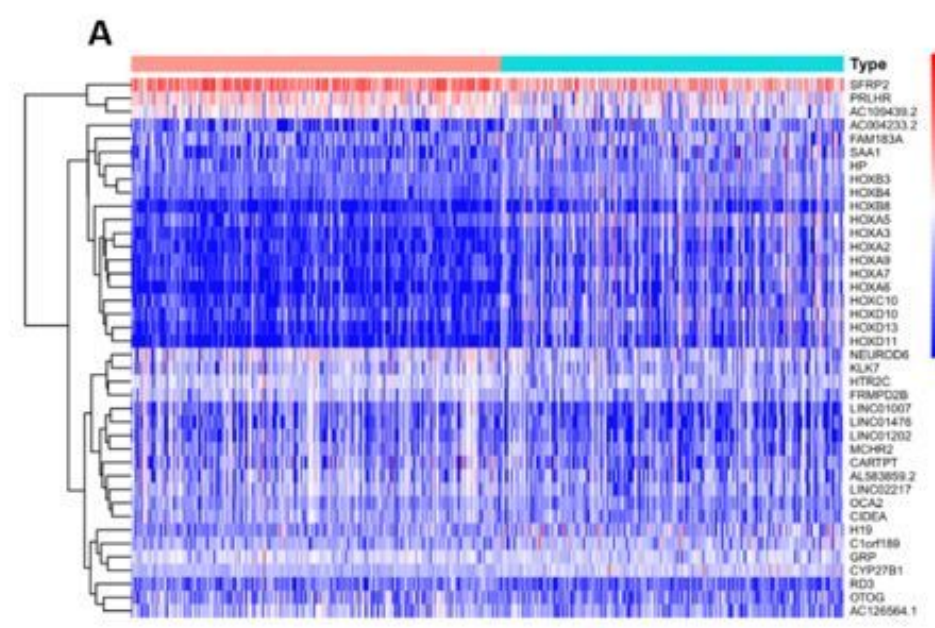

B

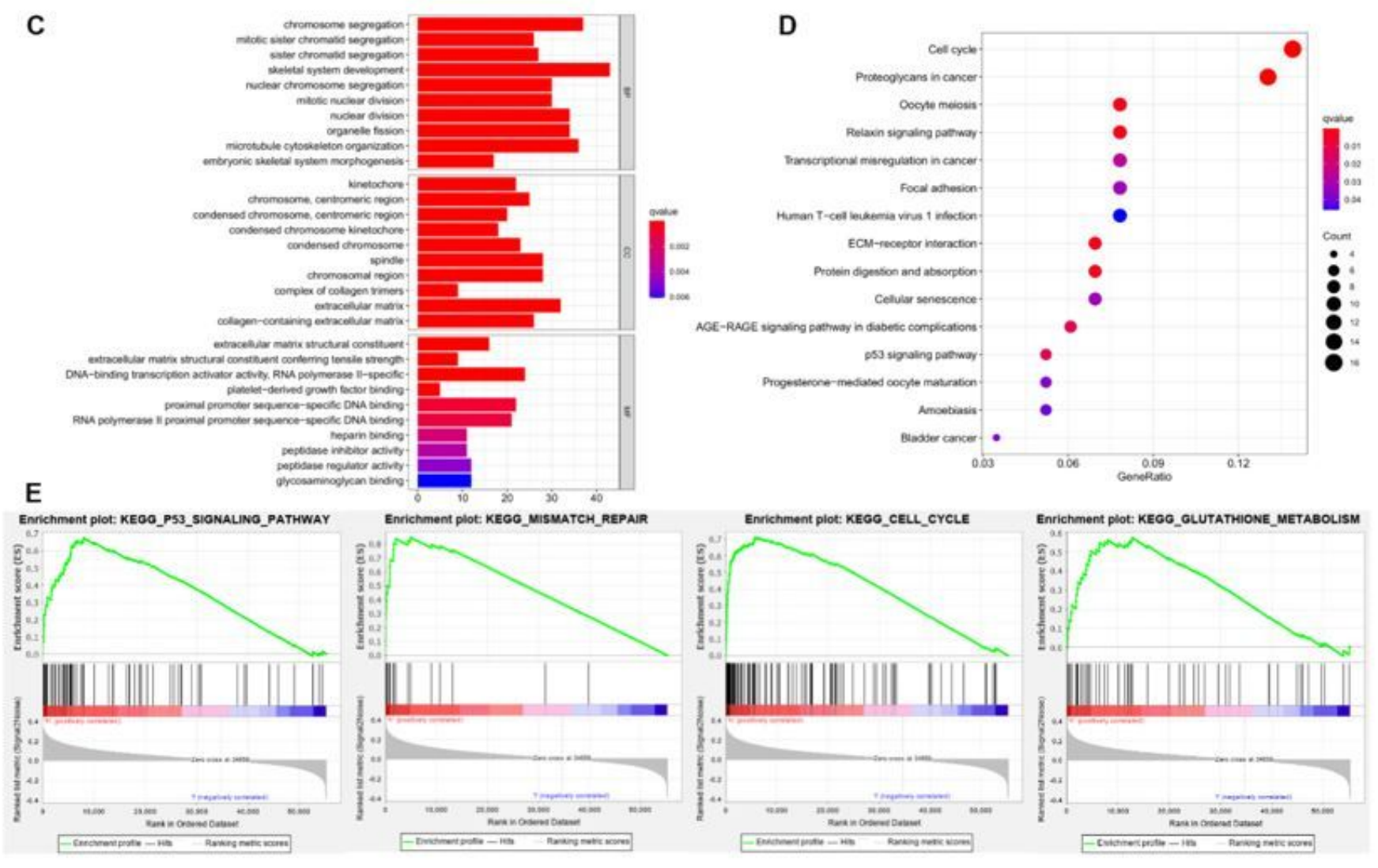

Figure 5

GO, KEGG and GSEA analysis (A) Heatmaps visualizing dissimilarity between high-TMB and low-TMB groups. (B) Venn diagrams of TMB-related immune genes. (C) GO enrichment bar plot. (D) KEGG enrichment bubble plot. (E) Parts of GSEA results. 




- B cells naive

- B cells memory

- Plasma cels

- T cells COB

- Tceils CDa naive

- Trets CDa menory resting

T cells CDA memory activated

- T cells regulatory (Tregs)

- T cells regulatory (Treps)

- NK cells resting

NK cells activated

- Monocytes

- Macrophages MO

- Macrophages MY

- Macrophages M2

- Dendrisic cells activaled

- Mast cels resting

Mast cels activated

Eosinophis

B

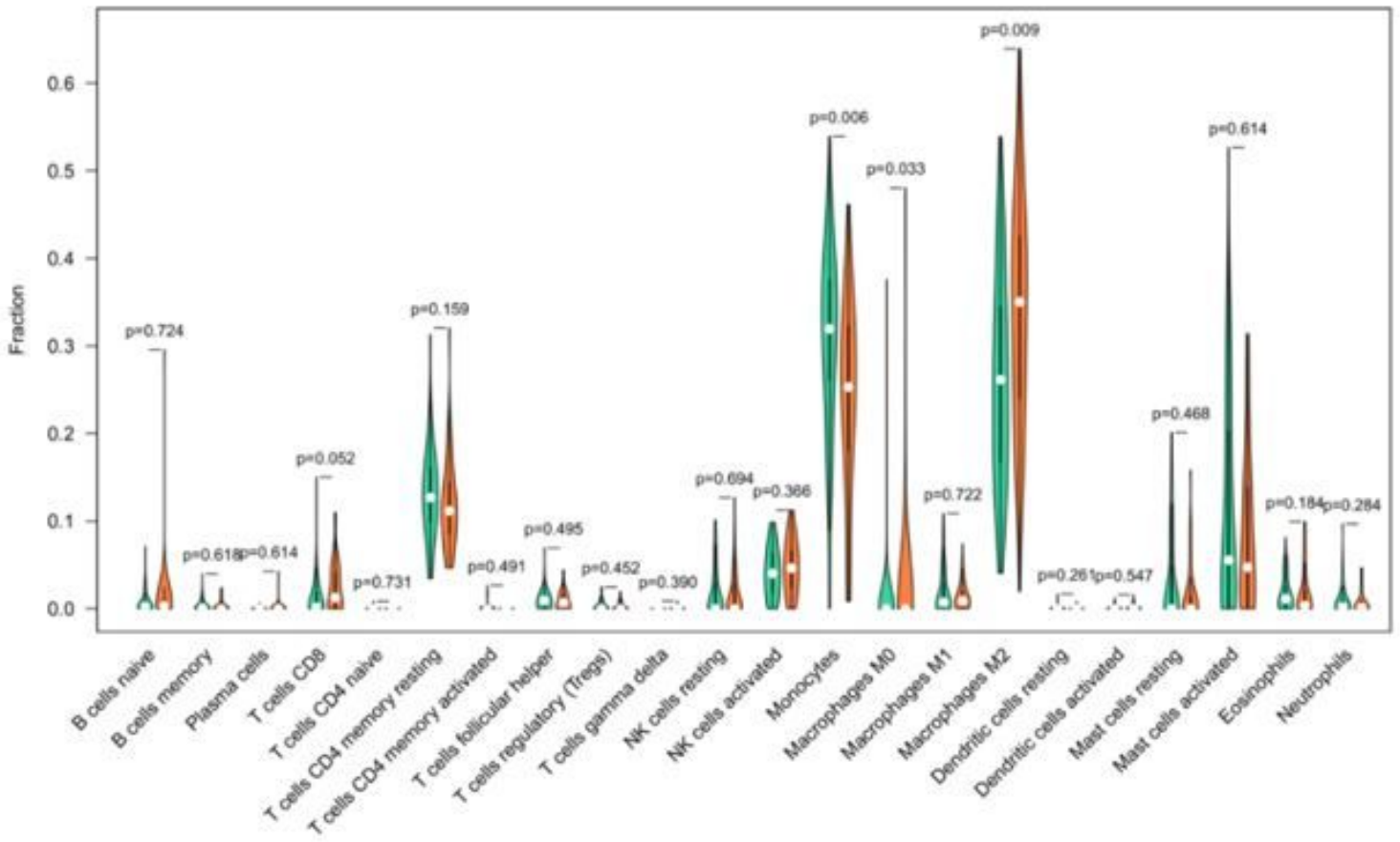

Figure 6

CIBERSORT results (A) Proportions of 22 immune cell subpopulations in lower-grade glioma analyzed by CIBERSORT (B) Comparisons of immune cells infiltration between high-TMB and low-TMB groups using CIBERSORT results. The orange column indicates the high-TMB group and the green column indicates the low-TMB group. 


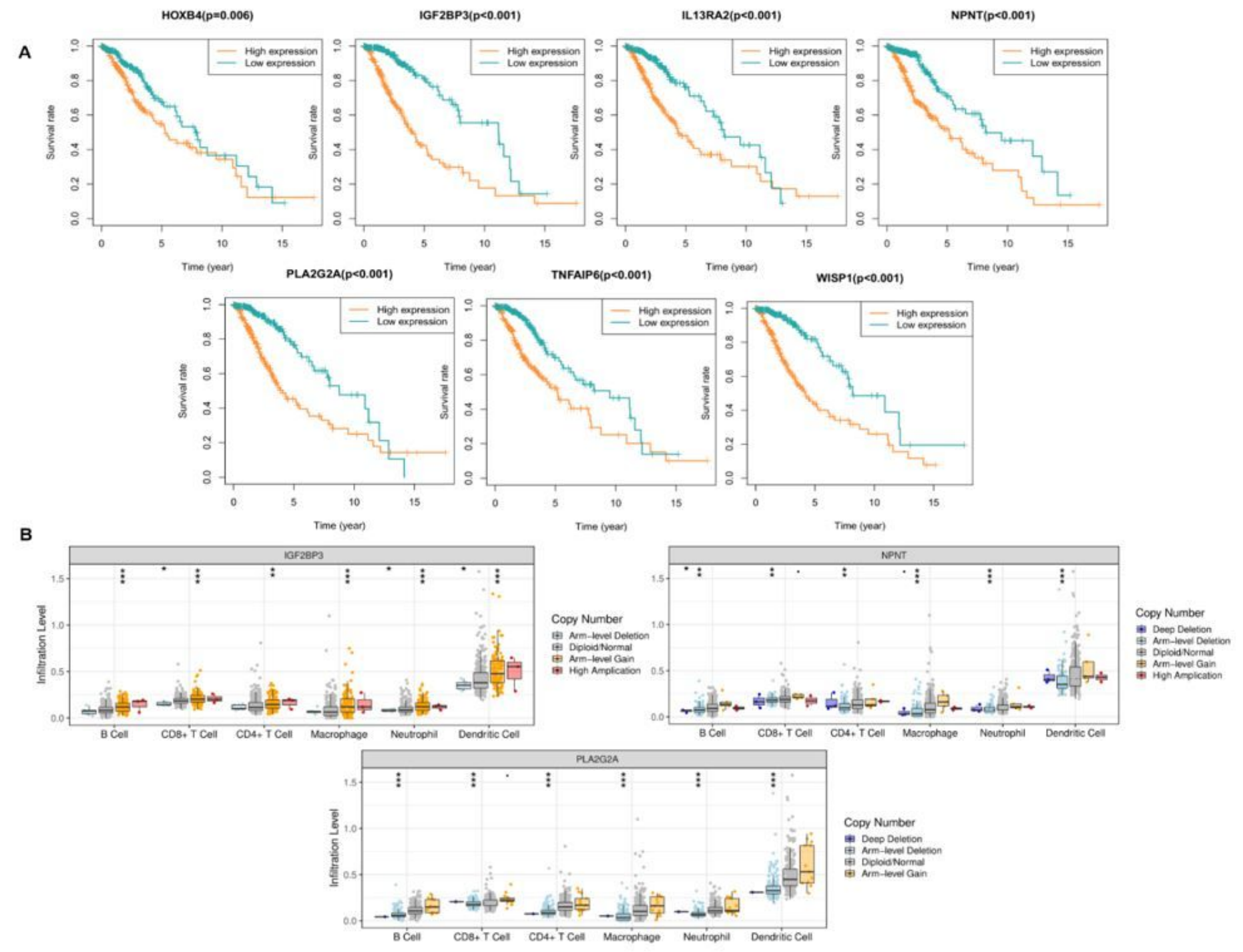

Figure 7

(A) Survival analysis of 7 hub immune genes. (B) SCNA of PLA2G2A, IGF2BP3 and NPNT have significant impacts on immune cells infiltration. Codes above various immune cells mean that different kinds of SCNAs affect the corresponding immune cells significantly. P-value significant codes: $0 \leq \star \star \star<$ $0.001 \leq * *<0.01 \leq *<0.05 \leq .<0.1$. 
A Survival curve(TCGA, $p<0.001)$
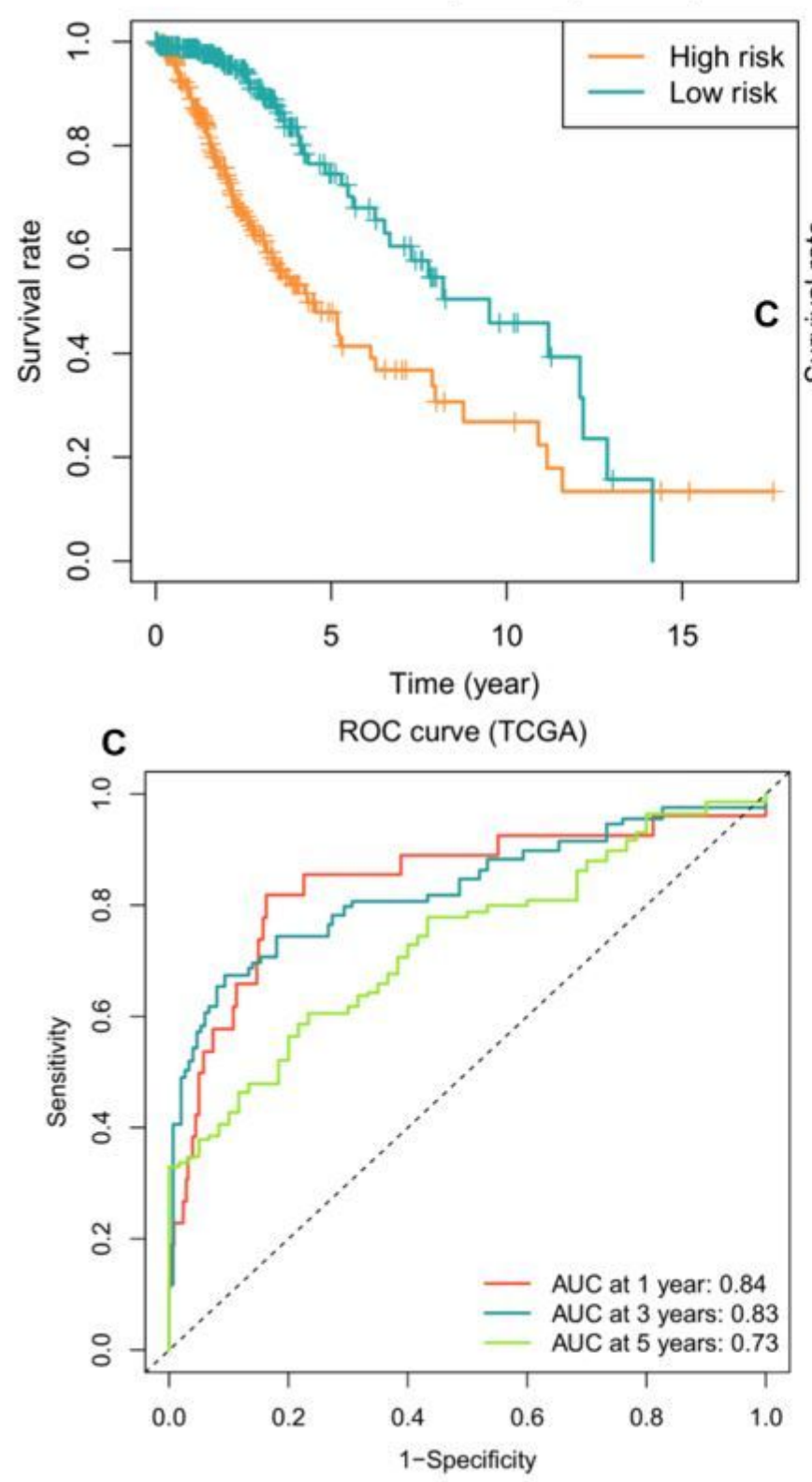

B Survival curve(CGGA, $p<0.001)$
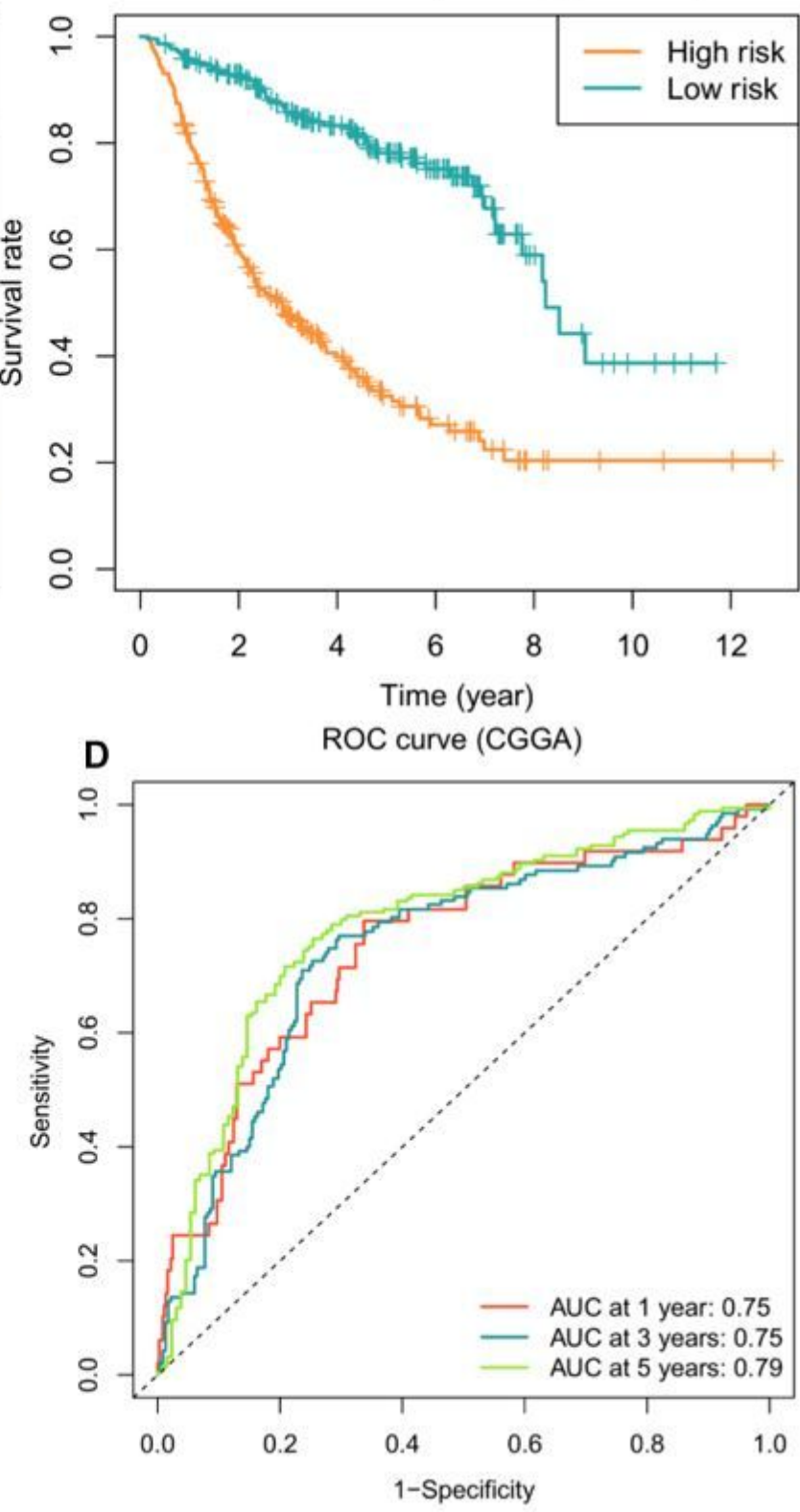

Figure 8

TMBPI prediction model for lower-grade glioma. (A-B) Differences in overall survival between high- and low-risk groups by the median of TMBPI using TCGA and CGGA databases respectively. (C-D) ROC curves for TMBPI model using TCGA and CGGA databases respectively with AUC at 1-, 3- and 5-years.

\section{Supplementary Files}

This is a list of supplementary files associated with this preprint. Click to download.

- CGGAmRNAseq693.xlsx

- DEGs.xIsx 
- GSEAlowTMB.xls

- KEGG.xIsx

- GSEAhighTMB.xls

- IGF2BP3.xIsx

- GO.xIsx

- Supplementarymaterials.docx 